\title{
Hepatolithiasis as a Cause of Recurrent Cholangitis
}

\author{
Begoña Teresa Ochoa Villalabeitia, María Carmen González Serrano, Alba Vazquez Melero, \\ Maria Inmaculada Concepción Cruz González, Sandra Ruiz Carballo, and Silvia García Herrero
}

\begin{abstract}
Background: Hepatolithiasis is the presence of calculi in the intra-hepatic bile ducts. If left untreated, it may cause recurrent cholangitis, hepatic abscesses, cirrhosis, liver atrophy, or degeneration to cholangiocarcinoma. Morbidity increases in bilateral forms of presentation. It is thought that a transitory bactibilia stimulates the formation of lithiasis. This leads to a persistent bactibilia, and therefore increases the risk of cholangitis. The most commonly found micro-organisms in bile cultures are Escherichia coli, Klebsiella, Pseudomonas, and Proteus.

Case Presentation: We present the case of a 67-year-old male with a finding of portal hypertension and bilateral hepatolithiasis discovered while studying a dissociated cholestasis. After several unsuccessful attempts for complete extraction of hepatolithiasis through conservative means (transparietohepatic approach, endoscopic retrograde cholangiopancreatography with endoscopic laser therapy) and because of recurrent episodes of cholangitis, a simultaneous surgical and endoscopic approach was undertaken (cholecystectomy with bile duct exploration and intra-operative laser therapy). The patient currently remains asymptomatic.

Conclusion: The treatment of hepatolithiasis has evolved because of the increase in the number of non-surgical techniques such as endoscopic laser therapy. Currently a surgical approach implying cholecystectomy and bile duct exploration combined with endoscopic laser techniques could be the elective treatment option, allowing the prevention of hepatectomy especially in patients at elevated surgical risk and in bilateral cases of hepatolithiasis.
\end{abstract}

Keywords: endoscopic retrograde cholangiopancreatography; hepatolithiasis; magnetic resonance cholangiopancreatography; recurrent cholangitis

$\mathbf{T}$ HIS IS THE CASE of an asymptomatic 67-year-old male with type 2 diabetes mellitus. He had undergone a laparoscopic right hemicolectomy in 2005 with posterior chemotherapy and without current treatment.

The patient presented for a general surgery consultation after being found to have bilateral hepatolithiasis by the gastroenterologist studying a dissociated cholestasis. The abdominal ultrasound examination showed multiple hyperechogenic images with acoustic posterior shadowing (Fig. 1). The computed tomography (CT) scan and magnetic resonance imaging (MRI) examination revealed signs of chronic hepatopathy with portal hypertension, dilation of the left intra-hepatic biliary ducts and of the posterior right segment of biliary ducts caused by several hepatolithiasis (Fig. 2).

Because the patient was asymptomatic, follow-up took place with periodic blood examinations. After discussion during the hepatobiliary surgery session, treatment onset was decided. Because of the elevated patient surgical risk, a surgical approach such as hepatectomy was rejected. It was decided to perform initially a transparietohepatic extraction of lithiasis. This procedure confirmed a bilateral hepatolithiasis and some calculi were pushed from the right biliary ducts to the duodenum with a balloon catheter. In the left bile ducts, a large repletion defect along with important dilation of the ducts proximally were found; no lithiasis were able to be extracted in this location. An internal-external drainage catheter was placed (Fig. 3). For completion of treatment, using programmed endoscopic retrograde cholangiopancreatography (ERCP) combined with laser therapy, the prosthesis was removed and lithiasis fragmentation was performed. Using a choledochoscope the endoscopist trespassed the large lithiasis of the left bile duct. Because extraction with balloon and Dormia basket

Hospital Universitario Basurto, Bilbao, Spain.

(C) Begoña Teresa Ochoa Villalabeitia et al. 2017; Published by Mary Ann Liebert, Inc. This Open Access article is distributed under the terms of the Creative Commons License (http://creativecommons.org/licenses/by/4.0), which permits unrestricted use, distribution, and reproduction in any medium, provided the original work is properly credited. 


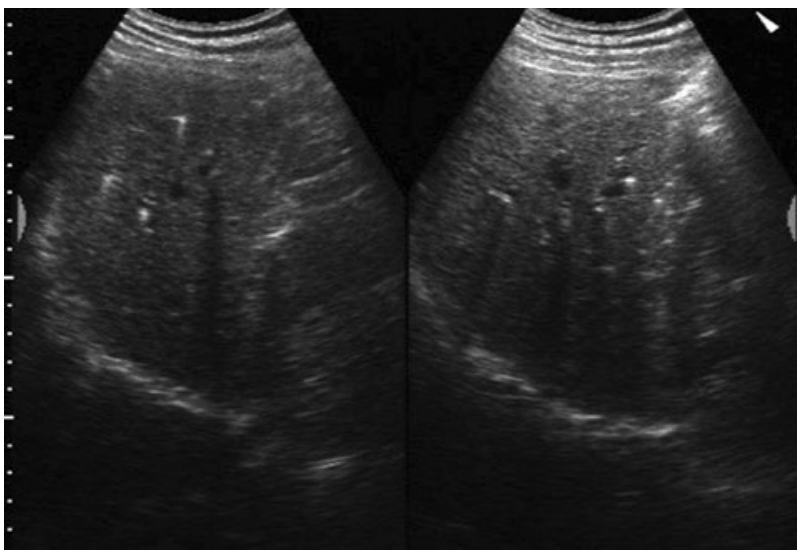

FIG. 1. Abdominal ultrasound view revealing multiple hyperechogenic images with acoustic posterior shadowing in the liver.

catheters was incomplete, a new $15-\mathrm{cm}$ plastic endoprosthesis was placed to drain the left bile ducts. It was decided that further extraction would be performed in a posterior programmed approach.

After discharge, the patient was re-admitted because of biliary septic shock (defined according to the new definitions of the Surviving Sepsis Guidelines) and required intensive care for seven days. The CT examination found a 2-cm diameter hepatic abscess (Fig. 4). After intravenous broadspectrum antibiotic agents (piperacilin/tazobactam) and fluid resuscitation, the prosthesis was removed urgently by endoscopy. Hemocultures were positive for Pseudomonas aeruginosa. After resolution of the episode and 48 hours after discharge, the patient was again re-admitted with cholangitis, which was resolved with the placement of a new internalexternal biliary drainage catheter and antibiotic agents. Because of the recurrent clinical setting, a programmed surgery with a simultaneous surgical and endoscopic approach was planned.

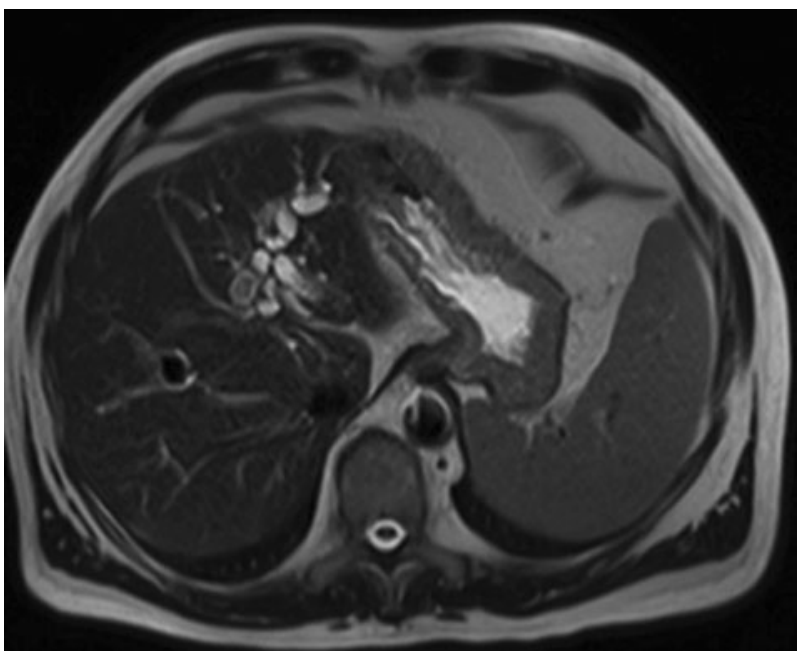

FIG. 2. Axial computed tomography (CT) view revealing dilatation of the left intra-hepatic bile ducts and of the right posterior segment of intra-hepatic bile ducts because of several hepatolithiasis, the largest being $1 \mathrm{~cm}$ in diameter.

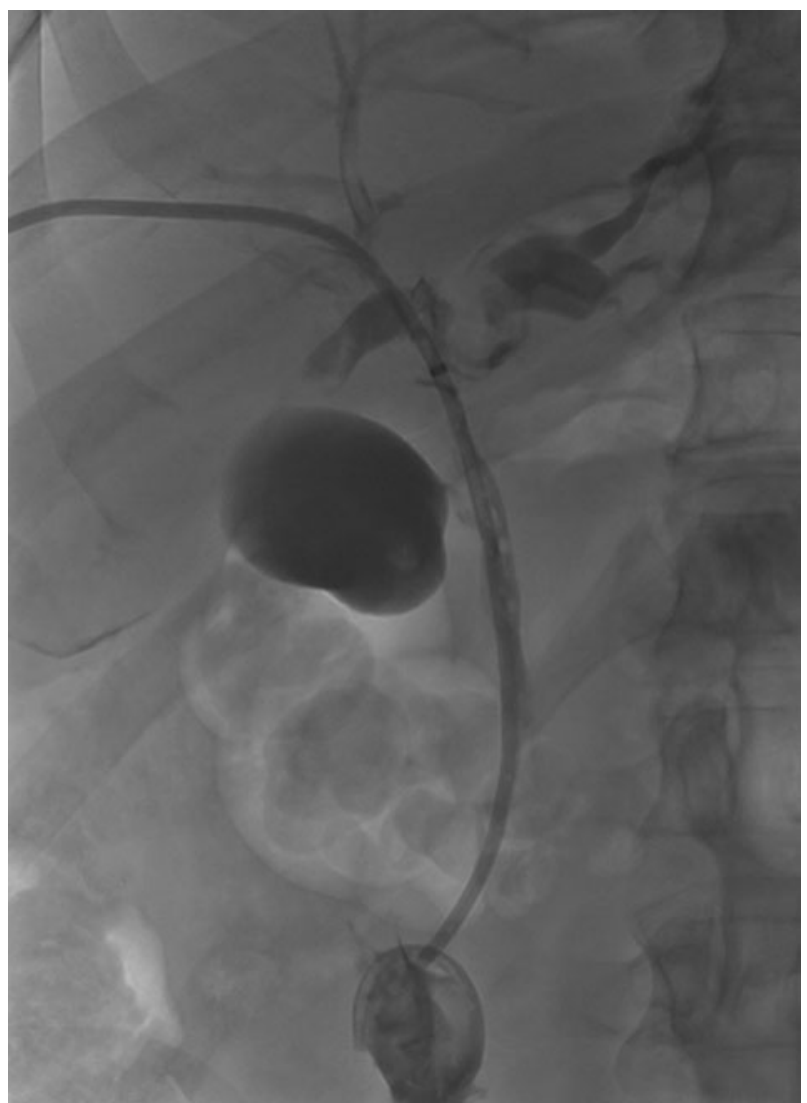

FIG. 3. Transparietohepatic cholangioscopic view showing the placement of an internal-external biliary drainage catheter.

The patient underwent an open cholecystectomy with intra-operative magnetic resonance cholangiopancreatography (MRCP), choledochotomy, and bile duct exploration with a choledochoscope. The large left hepatic duct lithiasis was fragmented with endoscopic laser therapy and extraction was performed with Dormia and Fogarty catheters. The

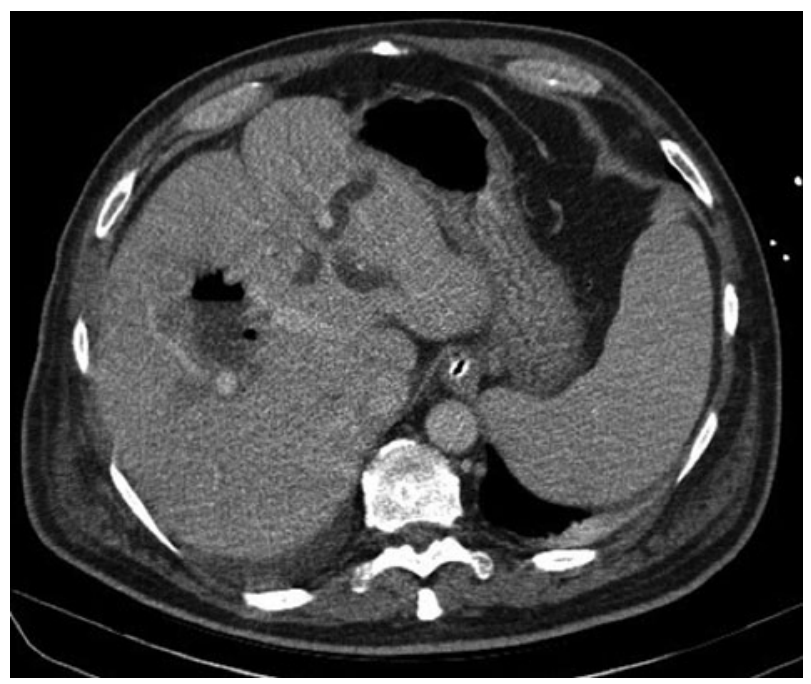

FIG. 4. Axial computed tomography (CT) view showing a 2-cm diameter hepatic abscess, with gas and liquid content. 
choledochotomy was closed over a Kehr tube. Discharge took place 22 days later. In consultations with hepatobiliary surgery, the Kehr tube was removed. Follow-up showed clinical improvement with decrease in bilirubin and transaminase levels. The patient overcame a new episode of autolimited cholangitis and currently remains asymptomatic.

\section{Discussion and Literature Review}

Hepatolithiasis is the presence of intra-hepatic bile duct lithiasis [1]. It is prevalent in East Asia but its prevalence is increasing in the Western countries because of immigration $(0.6 \%-1.3 \%)[2,3]$. Hepatolithiasis is known as the main cause of recurrent pyogenic cholangitis. If left untreated, it may lead to chronic liver disease resulting in high morbidity [4].

In infected bile, the typical pathogens found in cultures are the gram-negative enteric aerobes such as Escherichia coli, Klebsiella spp., and Proteus spp. [5]. It is known that any procedure that implies manipulation of the bile ducts increases risk of bactibilia and therefore infectious risk. In a study by González et al. at our hospital, it was demonstrated that patients who underwent bile duct decompression procedures presented positive bile cultures in more than $80 \%$ of the cases.

Biliary prostheses may become occluded by bacterial biofilms, especially if plastic is the material used. In case of cholangitis urgent removal is mandatory. Despite infection, the longer they remain, the more resistant the micro-organisms selected will be. One of the most frequently related bacteria in these cases is Pseudomonas aeruginosa [6], which bases its mechanisms of resistance in the creation of biofilms.

Currently several treatment options for hepatolithiasis are available. Among the non-surgical techniques include percutaneous transhepatic cholangioscopy and ERCP. These are used preferentially in bilateral lithiasis and in poor surgical candidates for hepatectomy or after unsuccessful techniques. They may be combined with laser lithotripsy when fragmentation is difficult. Frequently multiple sessions are required to allow complete extraction of lithiasis. Recently it has been shown that a combined surgical and endoscopic laser approach allows a satisfactory extraction of intrahepatic bile duct lithiasis avoiding liver resection, especially in the case of bilateral hepatolithiasis and in patients with elevated risk for hepatectomy [7].

\section{Conclusion}

The treatment of hepatolithiasis has evolved. A combined surgical approach with intra-operative endoscopic laser therapy may be a successful option in bilateral intra-hepatic biliary lithiasis, especially in high surgical risk patients in order to avoid hepatectomy.

\section{Author Disclosure Statement}

No competing financial interests exist.

\section{References}

1. Jarufe N, Figueroa E, Muñoz C, et al. Anatomic hepatectomy as a definitive treatment for hepatolithiasis: A cohort study. HPB (Oxford) 2012;14:604-610.

2. Kayhan B, Akdogan M, Parlak E, et al. Hepatolithiasis: A Turkey experience. Turk J Gastroenterol 2007;18:28-32.

3. Li C, Wen T. Surgical management of hepatolithiasis: A minireview. IRDR 2017;6:102-105.

4. Cook J, Hou P, Ho H, et al. Recurrent pyogenic cholangeitis. Br J Surg 1954;42:188-203.

5. Morris-Stiff GJ, O’Donohue P, Ogunbiyi S, Sheridan WG. Microbiological assessment of bile during cholecystectomy: Is all bile infected? HPB (Oxford) 2007;9:225-228.

6. Rodriguez Guardado A, Maradona JA, Carton JA, et al. Pseudomonas aeruginosa bacteremia as a complication after endoscopic retrograde cholangiopancreatography [in Spanish]. Enferm Infecc Microbiol Clin1997;15:540-536.

7. Tejera Hernandez AA, Cabrera Garcia ME, Navarro Medina $\mathrm{P}$, et al. Hepatolithiasis. Surgical approach using endoscopic holmium laser for treatment. Gastroenterol Hepatol 2017;40: 16-18.

Address correspondence to: Dr. Begoña Teresa Ochoa Villalabeitia

C/Juan Barroeta 8-A Neguri, Getxo Spain

E-mail: begona.ochoavillalabeitia@osakidetza.eus

Abbreviations Used
$\mathrm{CT}=$ computed tomography
$\mathrm{ERCP}=$ endoscopic retrograde
cholangiopancreatography
$\mathrm{MRCP}=\begin{gathered}\text { magnetic resonance } \\ \text { cholangiopancreatography }\end{gathered}$
$\mathrm{MRI}=$ magnetic resonance imaging

Cite this article as: Villalabeitia BTO, Serrano MCG, Melero AV, González MICC, Carballo SR, Herrero SG (2017) Hepatolithiasis as a cause of recurrent cholangitis. Surgical Infections Case Reports 2:1, 113-115, DOI: 10.1089/crsi.2017.0027 